\title{
A Hofer-Type Norm of Hamiltonian Maps on Regular Poisson Manifold
}

\author{
Dawei Sun ${ }^{1}$ and Zhenxing Zhang ${ }^{2}$ \\ ${ }^{1}$ College of Science, Henan University of Technology, Zhengzhou 450001, China \\ ${ }^{2}$ School of Mathematical Sciences, Nankai University, Tianjin 300071, China \\ Correspondence should be addressed to Dawei Sun; sundawei16@163.com
}

Received 17 November 2013; Accepted 8 February 2014; Published 27 March 2014

Academic Editor: Jin L. Kuang

Copyright (c) 2014 D. Sun and Z. Zhang. This is an open access article distributed under the Creative Commons Attribution License, which permits unrestricted use, distribution, and reproduction in any medium, provided the original work is properly cited.

We define a Hofer-type norm for the Hamiltonian map on regular Poisson manifold and prove that it is nondegenerate. We show that the $L^{1, \infty}$-norm and the $L^{\infty}$-norm coincide for the Hamiltonian map on closed regular Poisson manifold and give some sufficient conditions for a Hamiltonian path to be a geodesic. The norm between the Hamiltonian map and the induced Hamiltonian map on the quotient of Poisson manifold $(M,\{\cdot, \cdot\})$ by a compact Lie group Hamiltonian action is also compared.

\section{Introduction and Main Results}

This paper is devoted to establishing an invariant norm for Hamiltonian maps on the Poisson manifold. When $M$ is symplectic, a remarkable bi-invariant distance was defined on $\operatorname{Ham}(M)$. This bi-invariant distance was first discovered by Hofer on the group of compactly supported symplectic diffeomorphisms of $\left(\mathbb{R}^{2 n}, \omega_{0}\right)$ (where $\omega_{0}$ is the standard symplectic form) [1]. Viterbo defined a bi-invariant metric by generating functions [2], Polterovich generalized Hofer's metric to more symplectic manifold [3], and finally Lalonde and McDuff extended it to the group of compactly supported Hamiltonian diffeomorphisms on any symplectic manifold [4]. This norm plays an important role in studying symplectic topology and has close relationship with symplectic capacity and symplectic rigidity; many mathematicians have great work in this field, but there is few work on the Poisson case; this is because the lack of variational formulation in the Poisson case, it is not easy to prove the nondegenerate. In this paper, we define a Hofer-type norm on a class of Poisson manifolds, that is, regular Poisson manifolds; with the help of Casimir functions and the decomposition of Poisson manifold, we can prove the nondegenerate. Let $(M,\{\cdot, \cdot\})$ be a Poisson manifold; that is, there exists a Poisson bracket $\{\cdot, \cdot\}$ on the smooth functions $C^{\infty}(M)$. For any $f, g, h \in C^{\infty}(M)$ it satisfies the following:
(1) $\{f, g\}=-\{g, f\}$,

(2) $\{f, g h\}=g\{f, h\}+h\{f, g\}$,

(3) $\{f,\{g, h\}\}+\{h,\{f, g\}\}+\{g,\{h, f\}\}=0$.

Definition 1. A smooth diffeomorphism $\varphi: M \rightarrow M$ is called a Poisson diffeomorphism if for all $g, h \in C^{\infty}(M)$, one has $\varphi^{*}\{g, h\}=\left\{\varphi^{*}(g), \varphi^{*}(h)\right\}$.

Given $h \in C^{\infty}(M)$, the Hamiltonian vector is defined by $X_{h}=\{r, h\}$. Let $\operatorname{Cas}(M)=\left\{f \in C^{\infty}(M):\{f, g\}=0\right.$, $\left.\forall g \in C^{\infty}(M)\right\}$ be the set of Casimir functions. In this paper, one considers the time-dependent Hamiltonian functions $C^{\infty}([0,1] \times M, R)$. If the manifold is compact, or the function is compactly supported, then the flow of the Hamiltonian vector globally exists. One denotes by $\mathscr{P}(M), \operatorname{Ham}(M)$ the set of such Hamiltonian flows and set of time-1 map of such flows, respectively.

For $f \in C^{\infty}(M)$, define

$$
\|f\|=\inf \left\{\left\|f_{1}\right\|_{\infty} \mid f=f_{1}+f_{2}, f_{2} \in \operatorname{Cas}(M)\right\} .
$$

If $H_{t}$ is a Hamiltonian flow with some Hamiltonian function $h_{t}(x)$, one defines its length to be

$$
\text { length }\left(H_{t}\right)=\int_{0}^{1}\left\|h_{t}(x)\right\| d t
$$


one can generalize the bi-invariant metric on $\operatorname{Ham}(M)$ to the Poisson case.

Definition 2. Now one can define the energy of $\phi \in \operatorname{Ham}(M)$,

$$
E(\phi)=\inf \left\{\text { length }\left(H_{t}\right) \mid\right.
$$

$H_{t}$ is a Hamiltonian flow ended with $\left.\phi\right\}$.

So one can define $d: \operatorname{Ham}(M) \times \operatorname{Ham}(M) \rightarrow[0, \infty)$

$$
d(\varphi, \psi)=E\left(\varphi^{-1} \circ \psi\right)
$$

for $\varphi, \psi \in \operatorname{Ham}(M)$.

Theorem 3. Let $(M,\{\cdot, \cdot\})$ be a regular Poisson manifold; the function $d$ is a bi-invariant metric; that is, for all $\varphi, \psi, \theta \in$ $\operatorname{Ham}(M)$ it satisfies the following:

(1) $d(\varphi, \psi) \geq 0$ and $d(\varphi, \psi)=0$ if and only if $\varphi=\psi$,

(2) $d(\varphi, \psi) \leq d(\varphi, \theta)+d(\theta, \psi)$ and $d(\varphi, \psi)=d(\psi, \varphi)$,

(3) $d(\theta \varphi, \theta \psi)=d(\varphi, \psi)=d(\varphi \theta, \psi \theta)$.

Here a Poisson manifold is called regular if the rank of the Poisson manifold is constant for all point. If one replaces the $L^{1, \infty}$-norm by the $L^{\infty}$-norm, one also gets a norm on $\operatorname{Ham}(M)$. One proves that they are equal on closed regular Poisson manifold.

Theorem 4. For $\phi \in \operatorname{Ham}(M)$ on a closed regular Poisson manifold, one has

$$
\|\phi\|_{1, \infty}=\|\phi\|_{\infty} .
$$

Let $(M,\{\cdot, \cdot\})$ be a Poisson manifold and let $G$ be a Lie group acting canonically, freely, and properly on $M$ via the map $\Phi: G \times M \rightarrow M$. Let $\mathscr{J}: M \rightarrow M / A_{G}^{\prime}$ be the corresponding optimal momentum map. Then the orbit space $M / G$ is a Poisson manifold with Poisson bracket $\{\cdot, \cdot\}^{M / G}$ uniquely characterized by the relation

$$
\{f, g\}^{M / G}(\pi(m))=\{f \circ \pi, g \circ \pi\}(m) ;
$$

for any $m \in M$ and $f, g: M / G \rightarrow R$ are arbitrary smooth functions.

For a $G$-invariant smooth function on $M$, the Hamiltonian flow $H_{t}$ of $X_{h}$ induces a Hamiltonian flow $H_{t}^{M / G}$, so one has a well-defined homomorphism

$$
\Psi: \operatorname{Ham}(M)^{G} \longrightarrow \operatorname{Ham}\left(\frac{M}{G}\right),
$$

where $\operatorname{Ham}(M)^{G}$ denotes the $G$-invariant Hamiltonian maps. Now one can give a similar result as stated in [5].

Theorem 5. For a G-invariant Hamiltonian path $F_{t}$ with Hamiltonian function $f(x)$, if $\inf _{g \in \operatorname{Cas}(M)}\|f-g\|=$ $\inf _{g \in \operatorname{Cas}^{G}(M)}\|f-g\|$, one has

$$
\left\|\Psi\left(F_{1}\right)\right\| \leq \text { length }\left(F_{t}\right) .
$$

Moreover, if the path is length-minimizing, one has the following corollary.
Corollary 6. If the G-invariant Hamiltonian path $F_{t}$ is lengthminimizing, then

$$
\left\|\Psi\left(F_{1}\right)\right\| \leq\left\|F_{1}\right\|
$$

Organization of This Paper. The organization is as follows. First we will introduce the definition of the distance and give some properties. Next we will show the proofs of Theorems 3 and 4 . Then we introduce the Poisson reduction. And last we give the proof of Theorem 5 .

\section{The Distance on $\operatorname{Ham}(M)$}

In this section, we recall the construction of Hofer-norm on $\operatorname{Ham}(M)$ and give our definition. For $h \in C^{\infty}(M)$, define $\|h\|_{\infty}=\max _{x \in M} h(x)-\min _{x \in M} h(x)$.

For $f \in C^{\infty}(M)$, we now define

$$
\|f\|=\inf \left\{\left\|f_{1}\right\|_{\infty} \mid f=f_{1}+f_{2}, f_{2} \in \operatorname{Cas}(M)\right\} .
$$

Proposition 7. $\|\cdot\|$ defined above is a pseudonorm.

Proof. We just need to show the triangle inequality holds. Let $f=f_{1}+f_{2}$ and $g=g_{1}+g_{2}$ such that $\left\|f_{1}\right\|_{\infty} \leq\|f\|+\epsilon$, and $\left\|g_{1}\right\|_{\infty} \leq\|g\|+\epsilon$ for given $\epsilon>0$. Then

$$
\begin{aligned}
\|f+g\| & \leq\left\|f_{1}+g_{1}\right\|_{\infty} \\
& \leq\left\|f_{1}\right\|_{\infty}+\left\|g_{1}\right\|_{\infty} \\
& \leq\|f\|+\|g\|+2 \epsilon .
\end{aligned}
$$

From the definition we can see that $\|h\|=0$ when $h \epsilon$ $\operatorname{Cas}(M)$, so together with the triangle inequality we get that if $f, g \in C^{\infty}(M)$ and satisfies $f-g \in \operatorname{Cas}(M)$, then $\|f\|=$ $\|g\|$.

Proposition 8. The new pseudonorm $\|\cdot\|$ is $\operatorname{Ham}(M)$ invariant.

Proof. First, note that $\|\cdot\|_{\infty}$ is $\operatorname{Ham}(M)$-invariant. If $f=f_{1}+$ $f_{2}$ then $f_{1} \circ \phi, f_{2} \circ \phi$ is a decomposition of $f \circ \phi$. So

$$
\begin{aligned}
\|f \circ \phi\| & \leq\left\|f_{1} \circ \phi\right\|_{\infty} \\
& \leq\left\|f_{1}\right\|_{\infty} .
\end{aligned}
$$

So $\|f \circ \phi\| \leq\|f\|$ for any $\phi \in \operatorname{Ham}(M)$, and $\|f\|=\| f \circ \phi \circ$ $\phi^{-1}\|\leq\| f \circ \phi \|$.

We get that $\|f \circ \phi\|=\|f\|$.

Now consider a Hamiltonian function $h_{t} \in C^{\infty}([0,1] \times$ $M, R)$; the length of the Hamiltonian path generated by $h_{t}$ can be defined as follows:

$$
\operatorname{length}\left(H^{[0,1]}\right)=\int_{0}^{1}\left\|h_{t}(x)\right\| d t \text {. }
$$

This length is well defined; that is, it is independent the choice of the Hamiltonian functions. This is because of our pseudonorm vanishing on $\operatorname{Cas}(M)$. 
Definition 9. Now one can define the energy of $\phi \in \operatorname{Ham}(M)$,

$$
E(\phi)=\inf \left\{\text { length }\left(H_{t}\right) \mid\right.
$$

$H_{t}$ is a Hamiltonian flow ended with $\left.\phi\right\}$.

Proposition 10. The energy function $E: \operatorname{Ham}(M) \rightarrow R$ has the following properties:

(1) $E(\varphi) \geq 0$ and $E(\varphi)=0 \Leftrightarrow \varphi=i d$,

(2) $E(\varphi)=E\left(\varphi^{-1}\right)$,

(3) $E\left(\vartheta \varphi \vartheta^{-1}\right)=E(\varphi)$,

(4) $E(\varphi \circ \psi) \leq E(\varphi)+E(\psi)$,

where $\varphi, \psi \in \operatorname{Ham}(M)$ and $\vartheta$ is a Poisson diffeomorphism of M.

To prove this, we need to investigate the Hamiltonian functions of the Hamiltonian flows. Similar to the symplectic case, for the symplectic case, see page 144 of [6].

Definition 11. If $h, k$ are smooth functions in $C^{\infty}([0,1] \times M, R)$ and $\vartheta \in \operatorname{Ham}(M)$ one defines the functions $\bar{h}, h \# k$ and $h_{\vartheta}$ as follows:

$$
\begin{aligned}
\bar{h}(t, x) & =-h\left(t, \varphi_{h}^{t}(x)\right), \\
(h \# k)(t, x) & =h(t, x)+k\left(t,\left(\varphi_{h}^{t}\right)^{-1}(x)\right), \\
h_{\vartheta}(t, x) & =h\left(t, \vartheta^{-1}(x)\right) .
\end{aligned}
$$

Proposition 12. If $h, k$ are smooth functions the following formulate hold true:

$$
\begin{gathered}
\varphi_{\bar{h}}^{t}=\left(\varphi_{h}^{t}\right)^{-1}, \\
\varphi_{h \# k}^{t}=\varphi_{h}^{t} \circ \varphi_{k}^{t}, \\
\vartheta \circ \varphi_{h}^{t} \circ \vartheta^{-1}=\varphi_{h_{\vartheta}}^{t}, \\
\left(\varphi_{h}^{t}\right)^{-1} \circ \varphi_{k}^{t}=\varphi_{g}^{t},
\end{gathered}
$$

where $g=\bar{h} \# k=(k-h)\left(t, \varphi_{h}^{t}\right)$.

To prove this, we need the following fact.

Lemma 13 (see [7]). If $\varphi$ is a Poisson map, and $f \in C^{\infty}([0,1] \times$ $M, \mathbb{R})$, then

$$
d \varphi \cdot X_{f \circ \varphi}=X_{f} \circ \varphi .
$$

Proof. For any function $g \in C^{\infty}([0,1] \times M, \mathbb{R})$, we have

$$
\begin{gathered}
X_{f \circ \varphi}[g \circ \varphi](z)=d g \circ \varphi(z) \cdot d \varphi\left(X_{f \circ \varphi}(z)\right), \\
X_{f}[g](\varphi(z))=d g(\varphi(z)) \cdot X_{f}(\varphi(z)) .
\end{gathered}
$$

Since $\varphi$ is a Poisson map, we have

$$
X_{g \circ \varphi} \varphi^{*} f=\{f \circ \varphi, g \circ \varphi\}=\{f, g\} \circ \varphi=\varphi^{*} X_{g} f .
$$

So we have

$$
d \varphi\left(X_{f \circ \varphi}(z)\right)=X_{f \circ \varphi}(z)
$$

Proof of Lemma 13. The third formula is just the transition law of Hamiltonian vector. We know that if $\varphi$ is a Poisson map then

$$
\begin{gathered}
\varphi_{*} X_{f \circ \varphi}=X_{f}(\varphi), \\
\frac{d}{d t} \vartheta \circ \varphi_{h}^{t} \circ \vartheta^{-1}=\vartheta_{*} X_{h_{t}} \circ \vartheta^{-1}=X_{h \circ \vartheta^{-1}}
\end{gathered}
$$

Now we prove the second formula; we abbreviate the notation and observe that

$$
\begin{aligned}
& \frac{d}{d t} \varphi^{t}=X_{h} \circ \varphi^{t}, \quad \varphi^{0}=i d, \\
& \frac{d}{d t} \psi^{t}=X_{h} \circ \psi^{t}, \quad \psi^{0}=i d .
\end{aligned}
$$

We need to show that $\varphi^{t} \circ \psi^{t}$ is the flow of $X_{h \# k}$,

$$
\begin{aligned}
\frac{d}{d t} \varphi^{t} \circ \psi^{t}= & \frac{d}{d t} \varphi^{t} \circ \psi^{t}+\left(d \varphi^{t} \circ \psi^{t}\right) \cdot \frac{d}{d t} \psi^{t} \\
= & X_{h}\left(\varphi^{t} \circ \psi^{t}\right)+\left[d \varphi^{t} \circ\left(\varphi^{t}\right)^{-1} \circ \varphi^{t} \circ \psi^{t}\right] \\
& \cdot X_{k} \circ\left[\left(\varphi^{t}\right)^{-1} \circ \varphi^{t} \circ \psi^{t}\right] \\
= & X_{h}\left(\varphi^{t} \circ \psi^{t}\right)+\left[d\left(\varphi^{t}\right)^{-1}\right]^{-1} \\
& \cdot X_{k} \circ\left[\left(\varphi^{t}\right)^{-1} \circ \varphi^{t} \circ \psi^{t}\right] \\
= & X_{h}\left(\varphi^{t} \circ \psi^{t}\right)+X_{k \circ\left(\varphi^{t}\right)^{-1}}\left(\varphi^{t} \circ \psi^{t}\right) \\
= & X_{h \# k}\left(\varphi^{t} \circ \psi^{t}\right) .
\end{aligned}
$$

By the property of the Poisson diffeomorphism, we get that the second term is $X_{k \circ\left(\varphi^{t}\right)^{-1}}$. This finishes the proof of the second formula. We can obtain the first formula from the second. From the first two we can get the last one.

We are now ready to prove Proposition 10.

Proof of Proposition 10. From Proposition 12 and the Haminvariant of our pseudonorm, we get

$$
\text { length }\left(\varphi_{h}^{t}\right)=\text { length }\left(\left(\varphi_{h}^{t}\right)^{-1}\right),
$$

and thus $E(\varphi)=E\left(\varphi^{-1}\right)$. From

$$
\vartheta \circ \varphi_{h}^{t} \circ \vartheta^{-1}=\varphi_{h_{\vartheta}}^{t},
$$

we find

$$
\operatorname{length}\left(\vartheta \circ \varphi_{h} \circ \vartheta^{-1}\right)^{[0,1]}=\operatorname{length}\left(\varphi_{h_{\vartheta}}\right)^{[0,1]},
$$

so the third equality holds. 
To prove the last one, we note that $\varphi_{h \# k}^{t}=\varphi_{h}^{t} \circ \varphi_{k}^{t}$, and

$$
\begin{aligned}
\operatorname{length}\left(\varphi_{h \# k}^{[0,1]}\right) & =\int_{0}^{1}\|h \# k\| d t \\
& \leq \int_{0}^{1}\left\|h_{t}\right\|+\left\|k_{t}\right\| d t \\
& =\operatorname{length}\left(\left(\varphi_{h}\right)^{[0,1]}\right)+\operatorname{length}\left(\left(\varphi_{k}\right)^{[0,1]}\right),
\end{aligned}
$$

so we have $E\left(\varphi_{h} \circ \varphi_{k}\right) \leq E\left(\varphi_{h}\right)+E\left(\varphi_{k}\right)$, and this implies the last one. Now we prove the first one; that is, $E(\varphi)=0$ implies $\varphi=$ $i d$. By definition, $E(\varphi)=\inf \left\{\operatorname{length}\left(H_{t}\right) \mid H_{t}\right.$ generates $\left.\phi\right\}$. Note that regular Poisson manifold is essentially a union of symplectic manifolds which fit together in a smooth way, so we denote by $P_{\alpha}$ the symplectic leaf of $(M,\{\cdot, \cdot\})$. The Hamiltonian vector field restricted to each leaf is just the Hamiltonian vector field generated by the restriction of the Hamiltonian function to the leaf. And the Hamiltonian flow keeps the symplectic leaf; that is, $\varphi_{h}^{t} P_{\alpha_{0}}=P_{\alpha_{0}}$, so we can consider the restriction of $\varphi$ to each leaf. For each Hamiltonian function $h$ generating $\varphi$, we denote by $\widetilde{h}$ the restriction of $h$ on $P_{\alpha_{0}}$; for any Casimir function $g$, the restriction of $g$ on each leaf is constant. Let $\|\cdot\|_{P_{\alpha_{0}}}$ be the Hofer norm on the symplectic leaf,

$$
\begin{aligned}
\|\widetilde{h}\|_{P_{\alpha_{0}}}= & \|\widetilde{h}-\tilde{g}\|_{P_{\alpha_{0}}} \\
= & \int_{0}^{1}\left[\max _{x \in P_{\alpha_{0}}} h(t, x)-g(t, x)\right] \\
& -\min _{x \in P_{\alpha_{0}}}[h(t, x)-g(t, x)] d t \\
\leq & \int_{0}^{1}\left[\max _{x \in M} h(t, x)-g(t, x)\right] \\
& -\min _{x \in M}[h(t, x)-g(t, x)] d t .
\end{aligned}
$$

Taking the infimum of $g$, we get

$$
\|\widetilde{h}\|_{P_{\alpha_{0}}} \leq\|h\|
$$

and hence

$$
\inf _{h \text { generats } \varphi}\|\tilde{h}\|_{P_{\alpha_{0}}} \leq \inf _{h \text { generats } \varphi}\|h\| .
$$

By the assumption of $E(\varphi)=0$, we get that $\inf _{h \text { generats } \varphi}\|\widetilde{h}\|_{P_{\alpha_{0}}}=0$, by the definition of Hofer metric, $\left.\varphi\right|_{P_{\alpha_{0}}}=i d$ and $\operatorname{so} \varphi=i d$.

Now we can define $d: \operatorname{Ham}(M) \times \operatorname{Ham}(M) \rightarrow[0, \infty)$

$$
d(\varphi, \psi)=E\left(\varphi^{-1} \circ \psi\right),
$$

for $\varphi, \psi \in \operatorname{Ham}(M)$. This distance has the following properties.

Theorem $3{ }^{\prime}$. Let $(M,\{\cdot, \cdot\})$ be a Poisson manifold; the function $d$ is a bi-invariant metric; that is, for all $\varphi, \psi, \theta \in \operatorname{Ham}(M)$ it satisfies the following:
(1) $d(\varphi, \psi) \geq 0$ and $d(\varphi, \psi)=0$ if and only if $\varphi=\psi$,

(2) $d(\varphi, \psi) \leq d(\varphi, \theta)+d(\theta, \psi)$ and $d(\varphi, \psi)=d(\psi, \varphi)$,

(3) $d(\theta \varphi, \theta \psi)=d(\varphi, \psi)=d(\varphi \theta, \psi \theta)$.

Proof. The proof of this theorem is a consequence of Proposition 10 .

Example 14. We consider the trivial Poisson manifold $M$; the Poisson bracket $\{\cdot, \cdot\}$ is always zero. In this situation, any function on $M$ is Casimir function, the Hamiltonian vector is always zero and the Hamiltonian diffeomorphism is only $i d$; the Hofer-norm of the Hamiltonian map is 0 . When the Poisson manifold is symplectic, that is, there is only one leaf, in this case the Hofer norm is just the one defined by Hofer.

Remark 15. Theorem 3 holds not only for regular manifold, but also for many other manifolds, for example, when the rank of the Poisson manifold is not zero, or the symplectic leaves are always open or always closed.

Next we can get an estimate for the commutators in $\operatorname{Ham}(M)$, denoted by

$$
[\varphi, \psi]:=\varphi \psi \varphi^{-1} \psi^{-1} .
$$

Claim 1. $E([\varphi, \psi]) \leq 2 \min \{E(\varphi), E(\psi)\}$ for $\varphi, \psi \in \operatorname{Ham}(M)$.

Proof. From Proposition 10 we get

$$
\begin{aligned}
E([\varphi, \psi]) & =E\left(\varphi \psi \varphi^{-1} \psi^{-1}\right) \\
& \leq E\left(\varphi \psi \varphi^{-1}\right)+E\left(\psi^{-1}\right) \\
& \leq 2 E(\psi) .
\end{aligned}
$$

Similarly,

$$
\begin{aligned}
E([\varphi, \psi]) & =E\left(\varphi \psi \varphi^{-1} \psi^{-1}\right) \\
& \leq E(\varphi)+E\left(\psi \varphi^{-1} \psi^{-1}\right) \\
& \leq 2 E(\varphi),
\end{aligned}
$$

so the claim is proved.

Proposition 16. If $U \subset M$ is open and bounded, $\vartheta \in$ $\operatorname{Ham}(M)$ satisfies $\vartheta(U) \cap U=\emptyset$, then

$$
E([\varphi, \psi]) \leq 4 E(\vartheta)
$$

for all $\varphi, \psi \in \operatorname{Ham}(M)$ with supports contained in $U$

Proof. Define

$$
\gamma:=\varphi \vartheta^{-1} \varphi^{-1} \vartheta \in \operatorname{Ham}(M)
$$

since $\vartheta(U) \cap U=\emptyset$ and $\operatorname{supp}(\varphi) \subset U$, we get that $\left.\gamma\right|_{U}=\left.\varphi\right|_{U}$. Consequently

$$
\varphi \psi \varphi^{-1} \psi^{-1}=\gamma \psi \gamma^{-1} \psi^{-1}
$$


The above maps equal M. According to Claim 1 and Proposition 10 we get

$$
\begin{aligned}
E([\varphi, \psi]) & =E([\gamma, \psi]) \\
& \leq 2 E(\gamma) \\
& \leq 2 E\left(\vartheta^{-1}\right)+2 E(\vartheta) \\
& =4 E(\vartheta) .
\end{aligned}
$$

Proposition 17. For a subset $A \subseteq M$, define

$$
e(A)=\inf \{d(i d, \phi) \phi(A) \cap A=\emptyset, \phi \in \operatorname{Ham}(M)\},
$$

the displacement energy of $A$. Then for any open bounded nonempty subset $A$, one has

(1) $e(A) \neq 0$, if $A \subset B$, then $e(A) \leq e(B)$;

(2) $e(\phi(A))=e(A)$ for $\phi \in \operatorname{Ham}(M)$.

Proof. $e(A) \neq 0$ is a consequence of above statements and the monotonicity is by the definition. We just prove the second one. For $\phi, \theta \in \operatorname{Ham}(M)$, if $\theta(A) \cap A=\emptyset$, then $\phi \theta \phi^{-1} \phi(A) \cap$ $\phi(A)=\emptyset$; if $\theta \phi(A) \cap \phi(A)=\emptyset$, then $\phi^{-1} \theta \phi(A) \cap A=\emptyset$. According to Proposition 10, we have $E\left(\vartheta \varphi \vartheta^{-1}\right)=E(\varphi)$; from the above identities, we get the conclusion.

Following [8], we now define a new function for a Poisson map. For a Poisson map $\phi$ on a closed Poisson manifold $(M,\{\cdot, \cdot\})$, define

$$
r(\phi)=\sup \{E([\phi, f]) f \in \operatorname{Ham}(M)\},
$$

where $[\phi, f]=\phi f(\phi)^{-1} f^{-1}$. Similarly, we can define $r_{\alpha}(\phi)$ if we restrict $f$ in the closed ball of radius of $\alpha$ of $\operatorname{Ham}(M)$ centered at $i d$.

Definition 18. A map $\phi$ on a closed regular Poisson manifold $(M,\{\cdot, \cdot\})$ is bounded if $r(\phi)<+\infty$ and unbounded otherwise.

Proposition 19. For any $\alpha \in(0,+\infty]$, the function $r_{\alpha}(\phi)$ is bi-invariant, assumes the value 0 only at id, and satisfies the triangle inequality

$$
\begin{aligned}
r_{\alpha}(\phi \varphi) & \leq r_{\alpha}(\phi)+r_{\alpha}(\varphi), \\
r_{\alpha}(\phi) & \leq 2 \alpha,
\end{aligned}
$$

for any Poisson map $\phi$ and $\alpha \in(0,+\infty]$.

Proof. The proof is a consequence of the definitions of $r_{\alpha}$ and the metric.

Proposition 20. Let $(M,\{\cdot, \cdot\})$ be a closed regular Poisson manifold; if there exists some $\phi$ unbounded, then the Hofer metric d does not extend to a bi-invariant metric on the groups of Poisson maps.
Proof. Assume that we can extend the Hofer metric to the groups of Poisson maps; we still denote the metric by $d$; then $E([\phi, f])=E\left(\phi f(\phi)^{-1} f^{-1}\right) \leq 2 E(\phi)$ for any $f \in \operatorname{Ham}(M)$. So we have

$$
r(\phi) \leq 2 E(\phi),
$$

for any $\phi$, and this is impossible; hence we finish the proof.

Now we consider the geodesic under the above norm in $\operatorname{Ham}(M)$. For the standard symplectic manifold, Hofer proved that, the Hamiltonian flow generated by the timeindependent compactly supported function is a geodesic [6]. Later Bialy and Polterovich gave a sufficient and necessary condition for a path to be geodesic [9]; last Lalonde and McDuff extended it to all symplectic manifolds [4]. Now we consider similar questions on regular Poisson manifold.

Theorem 21. Let $\varphi_{h}^{t}$ be a Hamiltonian flow with compactly supported time-independent Hamiltonian function $h$ on $(M,\{\cdot, \cdot\})$, and if the maximal and minimal point of $h$ lie in the same symplectic leaf, then $\varphi_{h}^{t}$ is a geodesic; that is, $d\left(\varphi_{h}^{t}, \varphi_{h}^{s}\right)=$ $(t-s)\|h\|$ for $|s-t|$ sufficiently small.

Proof. First, by the definition we have

$$
d\left(\varphi_{h}^{t}, \varphi_{h}^{s}\right)=d\left(\varphi_{h}^{t-s}, i d\right) \leq|s-t|\|h\| .
$$

To prove the converse, let $P_{\alpha}$ be the symplectic leaves of the Poisson manifold; we still denote by $\widetilde{h}$ the restriction of $h$ on $P_{\alpha_{0}}$; for any compactly supported Hamiltonian function $g$ generating $\varphi_{h}^{t-s}$, we have

$$
\inf _{g \text { generats } \varphi_{h}^{t-s}}\|\tilde{g}\|_{P_{\alpha_{0}}} \leq \inf _{g \text { generats } \varphi_{h}^{t-s}}\|g\| .
$$

So

$$
\left\|\varphi_{h}^{t-s}\right\| \geq\left\|\varphi_{h}^{t-s}\right\|_{P_{\alpha_{0}}},
$$

on each leaf, but on each leaf, according to the results on symplectic manifold mentioned above, the flow $\varphi_{\tilde{h}}^{t}$ is a geodesic; we thus have $\left.\left\|\varphi_{h}^{t-s}\right\|\right|_{P_{\alpha_{0}}} \geq|s-t|\|\widetilde{h}\|$ for $|s-t|$ sufficiently small. By the assumption, we have

$$
\max _{x \in P_{\alpha_{0}}} h(x)-\min _{x \in P_{\alpha_{0}}} h(x) \geq \max _{x \in M} h(x)-\min _{x \in M} h(x),
$$

for some leaf $P_{\alpha_{0}}$. Hence

$$
\begin{array}{r}
\max _{x \in P_{\alpha_{0}}} h(x)-\min _{x \in P_{\alpha_{0}}} h(x) \geq \inf _{g \in \operatorname{Cas}(M)} \max _{x \in M} h(x)-g(x) \\
-\min _{x \in M} h(x)-g(x) .
\end{array}
$$

Since $h$ is compactly supported, we have the $C^{2}$-norm of $h$ is bounded above by some number $T$, and moreover,

$$
\|h\|_{C^{2}} \geq\left.\|h\|_{C^{2}}\right|_{P_{\alpha_{0}}},
$$

so by Theorem 1.2 of [3], we can choose a constant $T^{\prime}>T$ such that $\left\|\varphi_{h}^{t-s}\right\| \geq|s-t|\|h\|$ when $|s-t| \leq 1 / T^{\prime}$ and this finishes the proof. 
For the time-dependent case, we have a similar result. First we recall the definition of quasiautonomous function.

Definition 22. A function $h(t, x)$ on $[0,1] \times M$ is called quasiautonomous if there exist two points $x_{+}, x_{-} \in M$ such that $\max _{x} h(t, x)=h\left(t, x_{+}\right), \min _{x} h(t, x)=h\left(t, x_{-}\right)$for all $t \in[0,1]$.

Theorem 23. Let $\varphi_{h}^{t}$ be a Hamiltonian flow with compactly supported Hamiltonian function $h$ on $(M,\{\cdot, \cdot\})$; if h is quasiautonomous on each symplectic leaf, and the fixed maximum $x_{+}$ and fixed minimum $x_{-}$of h lie in the same symplectic leaf, then $\varphi_{h}^{t}$ is a geodesic; that is, $d\left(\varphi_{h}^{a}, \varphi_{h}^{b}\right)=\operatorname{Length}\left(\varphi_{h}^{[a, b]}\right)$ for $|a-b|$ sufficiently small.

Proof. We first adopt the transformation in [10] to simplify the problem; for interval $[a, b]$, define $\delta(t)=a+t(b-a)$ and $k(t, x)=(b-a) h(\delta(t), x)$ for $t \in[0,1], x \in M$. Then the Hamiltonian flow of $k(t, x)$ satisfies

$$
\begin{gathered}
\varphi_{k}^{t}=\varphi_{h}^{\delta(t)} \circ\left(\varphi_{h}^{a}\right)^{-1} \quad \text { for } t \in[0,1], \\
\varphi_{k}^{0}=i d, \quad \varphi_{k}^{1}=\varphi_{h}^{b} \circ\left(\varphi_{h}^{a}\right)^{-1}, \\
\int_{0}^{1} \inf _{g \in \operatorname{Cas}(M)}\left[\max _{x} k(t, x)-g(x)-\min _{x} k(t, x)-g(x)\right] d t \\
=\int_{a}^{b} \inf _{g \in \operatorname{Cas}(M)}\left\{\max _{x} h(t, x)-\frac{1}{b-a} g(x)\right. \\
=\int_{a}^{b} \inf _{g \in \operatorname{Cas}(M)}\left\{\max _{x} h(t, x)-g(x)\right. \\
\left.-\min _{x} h(t, x)-g(x)\right\} d t .
\end{gathered}
$$

This implies that $\|k\|=\|h\|$; by the assumption, $h(t, x)$ is quasiautonomous on each symplectic leaf and so is $k$; by Theorem 1.2 of $[11,12]$, we know that $\varphi_{k}^{t}$ is a minimum geodesic on each leaf provided that $|a-b|$ sufficiently small; that is,

$$
\left.d\left(\varphi_{k}^{0}, \varphi_{k}^{1}\right)\right|_{P_{\alpha_{0}}}=\left.\|k\|\right|_{P_{\alpha_{0}}}
$$

Because the fixed maximum $x_{+}$and fixed minimum $x_{-}$of $h$ lie in the same symplectic leaf, we may assume that this leaf is $P_{\alpha_{0}}$. We have

$$
\begin{aligned}
& \int_{0}^{1} \max _{x \in P_{\alpha_{0}}} h(t, x)-\min _{x \in P_{\alpha_{0}}} h(t, x) d t \\
& \quad \geq \int_{0}^{1} \max _{x \in M} h(t, x)-\min _{x \in M} h(t, x) d t .
\end{aligned}
$$

So,

$$
\begin{aligned}
& \int_{0}^{1} \max _{x \in P_{\alpha_{0}}} h(t, x)-\min _{x \in P_{\alpha_{0}}} h(t, x) d t \\
& \geq \int_{0}^{1} \inf _{g \in \operatorname{Cas}(M)}\left\{\max _{x \in M} h(t, x)-g(x)\right. \\
& \left.\quad-\min _{x \in M} h(t, x)-g(x)\right\} d t \\
& =\|h\| .
\end{aligned}
$$

By Theorem 3, we have $\|h\|=\|k\|=d\left(\varphi_{k}^{0}, \varphi_{k}^{1}\right)=d\left(\varphi_{h}^{a}, \varphi_{h}^{b}\right)$.

Remark 24. If the Poisson manifold is symplectic, then Theorems 21-23 reduce to the results in $[4,6,9]$. If we make more assumptions on the Hamiltonian functions, we can get similar results about the minimizing geodesics.

Theorem 25. Let $g_{i}, g$ be a sequence of smooth Hamiltonians on a closed Poisson manifold $M$; suppose that

(1) $g_{i} \rightarrow g$ in the $C^{0}$-topology,

(2) $\phi_{g_{i}}^{t} \rightarrow \phi_{g}^{t}$ in the $C^{0}$-topology, $t \in[0,1]$.

If all $\phi_{g_{i}}^{t}$ are minimizing, then $\phi_{g}^{t}$ is also minimizing.

Proof. We employ the method of Oh in [13] to prove it; for reader's convenience, we write it here. Suppose that $\phi_{g}^{t}$ is not minimizing, then we choose a function $f$, such that $\|f\|<$ $\|g\|$; choose $\delta>0$ such that

$$
\|f\|<\|g\|-\delta
$$

then

$$
\|f\|<\left\|g_{i}\right\|-\frac{\delta}{2}
$$

when $i$ is sufficiently large. Define

$$
f_{i}=g_{i}-g\left(\phi_{g_{i}}^{t}\right)+f\left(\phi_{g}^{t} \circ\left(\phi_{g_{i}}^{t}\right)^{-1}\right) \text {. }
$$

By simply computations as we know that $\phi_{f_{i}}^{1}=\phi_{g_{i}}^{1},\|f\|<$ $\left\|f_{i}\right\|-\delta / 2$ and $f_{i} \rightarrow f$ in the $C^{0}$-topology, so for any Casmir function $h$, we have $f_{i}-h \rightarrow f-h$, and thus

$$
\lim _{i \rightarrow \infty}\left\|f_{i}\right\|=\|f\| .
$$

This is a contraction and we finish the proof.

Theorem 26. Assume that $\phi_{i}$ and $\phi \in \operatorname{Ham}(M)$, and $\psi$ is a homeomorphism of $M$. If

(1) $d\left(\phi_{i}, \phi\right) \rightarrow 0$,

(2) $\lim _{i \rightarrow \infty} \phi_{i}=\psi$, locally uniformly,

then $\phi=\psi$. 
Proof. We assume that $\phi=i d$, and by the assumption we have $E(\phi) \rightarrow 0$ and $\lim _{i \rightarrow \infty} \phi_{i}=\psi$. We restrict them to each leaf and adapt the same notations and arguments in Proposition 10; we have $E\left(\left.\phi\right|_{P_{\alpha_{0}}}\right) \rightarrow 0$ and $\left.\lim _{i \rightarrow \infty} \phi_{i}\right|_{P_{\alpha_{0}}}=\left.\psi\right|_{P_{\alpha_{0}}}$. By Theorem 6 page 169 in [6], we know that $\left.\psi\right|_{P_{\alpha_{0}}}=i d$; this holds on each leaf, so $\phi=\psi$.

Corollary 27. If $\phi_{h_{i}} \in \operatorname{Ham}(M)$ and $\psi$ is a homeomorphism of $M$ satisfying

(1) $h_{i} \rightarrow$ h uniformly, $h_{t} \in \operatorname{Cas}(M)$

(2) $\lim _{i \rightarrow \infty} \phi_{i}=\psi$, locally uniformly

then $\psi=i d$.

If one replaces the $L^{1, \infty}$-norm by the $L^{\infty}$-norm, one also gets a pseudonorm on $\operatorname{Ham}(M)$.

For a Hamiltonian function $h \in C^{\infty}([0,1] \times M, R)$, define the pseudolength of the Hamiltonian path generated by $h$ as follows:

$$
\text { length }\left(H^{[0,1]}\right)=\max _{t \in[0,1]}\left\|h_{t}(x)\right\| \text {. }
$$

Similarly, one can define the energy and the pseudometric.

Definition 28. The energy of $\phi \in \operatorname{Ham}(M)$,

$$
E(\phi)=\inf \left\{\text { length }\left(H_{t}\right) \mid\right.
$$

$H_{t}$ is a Hamiltonian flow ended with $\left.\phi\right\}$.

Define $d: \operatorname{Ham}(M) \times \operatorname{Ham}(M) \rightarrow[0, \infty)$

$$
d(\varphi, \psi)=E\left(\varphi^{-1} \circ \psi\right),
$$

for $\varphi, \psi \in \operatorname{Ham}(M)$. This $d$ is also a bi-invariant metric. One denotes by $\|\cdot\|_{1, \infty},\|\cdot\|_{\infty}$ the induced $L^{1, \infty}$-norm and the $L^{\infty}$ norm, respectively.

Recall that in the symplectic case, Polterovich proved that the $L^{1, \infty}$-norm and the $L^{\infty}$-norm coincide on closed symplectic manifolds [14]. We now give a similar result in the Poisson case.

Theorem 4 . For $\phi \in \operatorname{Ham}(M)$ on a closed Poisson manifold, one has

$$
\|\phi\|_{1, \infty}=\|\phi\|_{\infty} .
$$

We first show the following results which will be useful in the proof.

Proposition 29. $\operatorname{Cas}(M)$ is closed in the $C^{0}$-topology.

Proof. We just show that this is true on each symplectic leaf, but in the symplectic case, the Casimir functions are constants; this finishes the proof.

Proposition 30. Let $F_{t}$ be a flow generated by a Hamiltonian function $f$ on a closed Poisson manifold. Then there exists an arbitrary small loop $H_{t}$ such that the Hamiltonian function $k_{t}$ of the flow $H_{t}^{-1} F_{t}$ satisfies $\{k(t), \cdot\} \neq 0$ for every $t$.
Proof. First, by Proposition 12 we know that the Hamiltonian function of $H_{t}^{-1} F_{t}$ can be given by $k(t, x)=f\left(t, H_{t} x\right)-$ $h\left(t, H_{t} x\right)$. We now just show that $\{k(t), \cdot\} \neq 0$ for all $t$. Take a function $g \in C^{\infty}([0,1] \times M, R)$ such that $\int_{0}^{1} g(t, x) d t=0$ for every $x \in M$. Then define $H_{t}(\epsilon) \in \operatorname{Ham}(M)$ as the time$\epsilon$ map of the Hamiltonian flow generated by $\int_{0}^{t} g(s, x) d s$. Let $h(t, \epsilon, x)$ be the Hamiltonian function of the loop $H_{t}(\epsilon)$, then we need the following proposition of Banyaga.

Proposition 31 (Banyaga, cf. Proposition 3.1.5 [15]). Let $h_{s, t}$ be a 2-family smooth parameters of diffeomorphisms on a smooth manifold $M$ such that $h_{0,0}=i d$. Let $X_{s, t}, Y_{s, t}$ be the families of vectors on $M$ defined by

$$
\begin{aligned}
& X_{s, t}(x)=\frac{d}{d t} h_{s, t}\left(h_{s, t}^{-1}(x)\right), \\
& X_{s, t}(x)=\frac{d}{d s} h_{s, t}\left(h_{s, t}^{-1}(x)\right) ;
\end{aligned}
$$

then

$$
\frac{\partial X_{s, t}}{\partial s}=\frac{\partial Y_{s, t}}{\partial t}+\left[X_{s, t}, Y_{s, t}\right]
$$

By the above proposition, we now compute $\left.(\partial / \partial \epsilon)\right|_{\epsilon=0} h(t, \epsilon, x)$. By definition

$$
\begin{aligned}
X_{\cdot, \epsilon} & =\left\{\cdot, h_{(\cdot, \epsilon)}\right\}, \\
Y_{t, \cdot} & =\left\{\cdot, h_{(t, \cdot)}\right\}, \\
\left.\frac{\partial X_{t, \epsilon}}{\partial \epsilon}\right|_{\epsilon=0} & =\left\{\cdot,\left.\frac{\partial h(t, \epsilon, x)}{\partial \epsilon}\right|_{\epsilon=0}\right\} \\
& =\left\{\cdot,\left.\frac{\partial h(t, \epsilon, x)}{\partial t}\right|_{\epsilon=0}\right\}+\left[X_{t, \epsilon}, Y_{t, \epsilon}\right] \\
& =\{\cdot, g(t, x)\} .
\end{aligned}
$$

So $\left.(\partial / \partial \epsilon)\right|_{\epsilon=0} h(t, \epsilon, x)=g(t, x)$ up to a Casimir function.

Now we define

$$
H_{t}\left(\epsilon_{1}, \ldots, \epsilon_{k}\right)=H_{t}^{1}\left(\epsilon_{1}\right) \circ \cdots \circ H_{t}^{k}\left(\epsilon_{k}\right) .
$$

Here every $H^{j}$ is constructed with the help of $g^{j}$ as above; we know that the partial derivative of the Hamiltonian $h(t, \epsilon, x)$ with respect to $\epsilon_{j}$ at $\epsilon=0$ equals $g^{j}$.

Fixed a regular point $y \in M$, let $N$ be the symplectic leaf though $y$, consider the linear space $E=T_{y} N \subseteq T_{y} M$. Choose $2 n$ smooth closed curves $\alpha_{1}(t), \ldots, \alpha_{2 n}(t)$ (where $t \in S^{1}$ ) satisfying the following conditions:

(1) $\int_{0}^{1} \alpha_{j}(t) d t=0$ for all $j=1, \ldots, 2 n$;

(2) the vectors $\alpha_{1}(t), \ldots, \alpha_{2 n}(t)$ are linearly independent for every $t$. The existence of such system of curves is shown in [14]; for example, choose a basis $u_{1}, v_{1}, \ldots, u_{n}, v_{n}$ in $E$ and take the curves of the form $u_{j} \cos 2 \pi t+v_{j} \sin 2 \pi t$ and $-u_{j} \sin 2 \pi t+v_{j} \cos 2 \pi t$. 
Now choose $\alpha_{j}(t), g_{j}(t)$ such that

(1) $g_{j}(t) \notin \operatorname{Cas}(M)$ for every $t$;

(2) $\int_{0}^{1} g_{j}(t, x) d t=0$ for every $x \in M$;

(3) $\left\{\cdot, g_{j}(t)\right\}(y)=\alpha_{j}(t)$.

Take the corresponding $2 n$-parameter variation $H_{t}(\epsilon)$ of the constant loop as above. Consider the map $\Gamma: S^{1} \times$ $R^{2 n}\left(\epsilon_{1}, \ldots, \epsilon_{2 n}\right) \rightarrow E$ defined by

$$
(t, \epsilon) \longrightarrow\left\{\cdot, f_{t}-h_{t}(\epsilon)\right\}(y) .
$$

It follows that $\Gamma$ is a submersion in some neighbourhood $U$ of the circle $\{\epsilon=0\}$. Indeed from our construction we have

$$
\left.\frac{\partial}{\partial \epsilon_{j}}\right|_{\epsilon=0} \Gamma(t, \epsilon)=\alpha_{j}(t)
$$

But these vectors generates the whole $E$. Denote by $\widetilde{\Gamma}$ the restriction of $\Gamma$ to $S^{1} \times U$. Since $\widetilde{\Gamma}$ is a submersion, the set $\widetilde{\Gamma}^{-1}(0)$ is a one-dimensional submanifold of $S^{1} \times U$, so there exist arbitrary small values of the parameter $\epsilon$ such that $\left\{\cdot, f_{t}-h_{t}(\epsilon)\right\}(y) \neq 0$ for all $t$. This completes the argument.

Proof of Theorem 4. For $\phi \in \operatorname{Ham}(M)$, clearly we have $\|\phi\|_{1, \infty} \leq\|\phi\|_{\infty}$. Now we prove the converse. Fix a positive number $\epsilon$, choose a path $F_{t} \in \operatorname{Ham}(M)$ such that $F_{0}=i d, F_{1}=$ $\phi$ with Hamiltonian functions $f_{t}$ and $\int_{0}^{1}\left\|f_{t}\right\| d t \leq\|\phi\|_{1, \infty}+\epsilon$. By Proposition 30 we can assume that $\left\|f_{t}\right\|>0$ for all $t$ since the manifold is good manifold. Here this pseudonorm is defined in Proposition 7. Define $a(t)$ as the inverse of

$$
b(t)=\frac{\int_{0}^{t}\left\|f_{t}\right\| d t}{\int_{0}^{1}\left\|f_{t}\right\| d t}
$$

where $t \in[0,1]$. Note that the Hamiltonian of $F_{a(t)}$ can be generated by $f^{a}=a^{\prime}(t) f(a(t), x)$, where $a^{\prime}(t)$ denotes the derivative with respect to $t$. Note that

$$
\max _{t \in[0,1]}\left\|a^{\prime}(t) f(a(t), x)\right\|=\max _{t \in[0,1]} \frac{\left\|f_{t}\right\|}{b^{\prime}(t)}=\int_{0}^{1}\left\|f_{t}\right\| d t
$$

we get that $\max _{t \in[0,1]}\left\|a^{\prime}(t) f(a(t), x)\right\| \leq\|\phi\|_{1, \infty}+\epsilon$. Approximating $a$ in the $C^{1}$-topology by a smooth one, we get a Hamiltonian denoted by $\tilde{f}$; we have $\max _{t \in[0,1]}\|\tilde{f}\|+2 \epsilon$. Since $\epsilon$ is arbitrary, we conclude that $\|\phi\|_{\infty} \leq\|\phi\|_{1, \infty}$. This completes the proof.

Corollary 32. For $f \in C^{\infty}([0,1] \times M, R)$, one has

$$
\left\|\phi_{f}^{1}\right\|_{1, \infty}=\left\|\phi_{f}^{1}\right\|_{\infty}=\inf \left\{\|f-h\|_{\infty} \mid \phi_{h}^{1}=i d\right\} .
$$

Proof. Let $\phi_{g}^{t}$ be another Hamiltonian flow with Hamiltonian $g(t, x)$ and satisfy $\phi_{g}^{1}=\phi_{f}^{1}$; then there exists a Hamiltonian function $h(t, x)$ such that $\phi_{g}^{t}=\phi_{h}^{t} \circ \phi_{f}^{t}$ and $\phi_{h}^{0}=\phi_{h}^{1}=i d$. On the other hand, for each loop $\phi_{h}^{t}$, the time one map of the flow $\phi_{h}^{t} \circ \phi_{f}^{t}$ is also $\phi_{f}^{1}$; by Proposition 12 its Hamiltonian can be

$$
g(t, x)=h(t, x)+f\left(t,\left(\phi_{h}^{t}\right)^{-1} x\right)
$$

Note that $\bar{h}=-h\left(t, \phi_{h}^{t} x\right)$ generates loop $\left(\phi_{h}^{t}\right)^{-1}$, so

$$
\left\|\phi_{f}^{1}\right\|_{1, \infty}=\inf \left\{\|f-h\|_{\infty} \mid \phi_{h}^{1}=i d\right\} .
$$

\section{Poisson Reduction}

In this section, we briefly introduce the Poisson reduction. Let $G$ be a Lie group acting canonically on $M$; if the action is free and proper, we know that the orbit space $M / G$ is a smooth manifold and the canonical projection $\pi: M \rightarrow M / G$ is a smooth surjective submersion. Let $\mathscr{J}: M \rightarrow M / A_{G}^{\prime}$ be the corresponding optimal momentum map. The orbit space $M / G$ is a Poisson manifold with Poisson bracket $\{\cdot, \cdot\}^{M / G}$ uniquely characterized by the relation

$$
\{f, g\}^{M / G}(\pi(m))=\{f \circ \pi, g \circ \pi\}(m),
$$

for any $m \in M$ and $f, g: M / G \rightarrow R$ are arbitrary smooth functions.

The Poisson structure induced by the bracket $\{\cdot, \cdot\}^{M / G}$ on $M / G$ is the only one for which the projection $\pi:(M,\{\cdot, \cdot\}) \rightarrow$ $\left(M / G,\{\cdot, \cdot\}^{M / G}\right)$ is a Poisson map. Let $h \in C^{\infty}(M)^{G}$ be a $G^{-}$ invariant Hamiltonian flow $F_{t}$ of $X_{h}$ commutes with the $G$ action, so it induces a flow $F_{t}^{M / G}$ on $M / G$ characterized by

$$
\pi \circ F_{t}=F_{t}^{M / G} \circ \pi \text {. }
$$

The flow $F_{t}^{M / G}$ is Hamiltonian on $\left(M / G,\{\cdot, \cdot\}^{M / G}\right)$ for the reduced Hamiltonian function $\widetilde{h} \in C^{\infty}(M / G)$ defined by

$$
\tilde{h} \circ \pi=h .
$$

The vector fields $X_{h}$ and $X_{\tilde{h}}$ are $\pi$-related.

So we have a well-defined homomorphism

$$
\Psi: \operatorname{Ham}(M)^{G} \longrightarrow \operatorname{Ham}\left(\frac{M}{G}\right)
$$

where $\operatorname{Ham}(M)^{G}$ denotes the $G$-invariant Hamiltonian maps.

More details can be found in [16].

\section{Proof of Theorem 5}

Now we can give the proof of Theorem 5.

Theorem 5'. For a G-invariant Hamiltonian path $F_{t}$ with Hamiltonian function $f$, if $\inf _{g \in \operatorname{Cas}(M)}\|f-g\|=$ $\inf _{g \in \operatorname{Cas}^{G}(M)}\|f-g\|$, one has

$$
\left\|\Psi\left(F_{1}\right)\right\| \leq \text { length }\left(F_{t}\right) .
$$


Proof. From the above discussion, we know that For any $G$ invariant Hamiltonian path $F_{t}$, it induces a Hamiltonian path $\Psi\left(F_{t}\right)$ on $\left(M / G,\{\cdot, \cdot\}^{M / G}\right)$.

Let $f \in C^{\infty}(M)^{G}, \tilde{f} \in C^{\infty}(M / G)$ be the Hamiltonian of the Hamiltonian path $F_{t}$, and the induced path $\Psi\left(F_{t}\right)$. We have

$$
\widetilde{f} \circ \pi=f
$$

By the definition of the norm, we have

$$
\sup _{x \in M / G} \widetilde{f(x)}-\inf _{x \in M / G} \widetilde{f(x)} \leq \sup _{x \in M} f(x)-\inf _{x \in M} f(x)
$$

and note that

$$
\inf _{g \in \operatorname{Cas}(M)}\|f-g\|=\inf _{g \in \operatorname{Cas}^{G}(M)}\|f-g\| .
$$

According to the above discussions, we have

$$
\begin{aligned}
\left\|\Psi\left(F_{1}\right)\right\| & \leq \operatorname{length}\left(\Psi\left(F_{t}\right)\right) \\
& =\inf _{g \in \operatorname{Cas}(M / G)}\|\tilde{f}-g\| \\
& \leq \inf _{g \in \operatorname{Cas}^{G}(M)}\|f-g\| \\
& \leq\|f(x)\|,
\end{aligned}
$$

so

$$
\left\|\Psi\left(F_{1}\right)\right\| \leq \text { length }\left(F_{t}\right) .
$$

Moreover, if the path is length-minimizing, that is, Length $\left(F_{t}\right)=\left\|F_{1}\right\|$ then we have Corollary 6 .

Remark 33. If the Poisson manifold is symplectic, then the pseudonorm is the Hofer norm. Give a Hamiltonian Gaction; then we can get the results in the symplectic case as in [5].

\section{Conflict of Interests}

The authors declare that they have no conflicts of interest regarding this work.

\section{Acknowledgments}

The authors would like to express their deep gratitude to Professor Yiming Long for many valuable discussions. The research was supported by TianYuan Program of National Natural Science Foundation of China (11226158), Natural Science Foundation of Henan (2011B110011), and Doctor Fund of Henan University of Technology.

\section{References}

[1] H. Hofer, "On the topological properties of symplectic maps," Proceedings of the Royal Society of Edinburgh. Section A, vol. 115, no. 1-2, pp. 25-38, 1990.

[2] C. Viterbo, "Symplectic topology as the geometry of generating functions," Mathematische Annalen, vol. 292, no. 4, pp. 685-710, 1992.

[3] L. Polterovich, "Symplectic displacement energy for Lagrangian submanifolds," Ergodic Theory and Dynamical Systems, vol. 13, no. 2, pp. 357-367, 1993.

[4] F. Lalonde and D. McDuff, "The geometry of symplectic energy," Annals of Mathematics, vol. 141, no. 2, pp. 349-371, 1995.

[5] A. Pedroza, "Induced Hamiltonian maps on the symplectic quotient," Differential Geometry and Its Applications, vol. 26, no. 5, pp. 503-507, 2008.

[6] H. Hofer and E. Zehnder, Symplectic Invariants and Hamiltonian Dynamics, Birkhäuser, Basel, Switzerland, 1994.

[7] J. E. Marsden and T. S. Ratiu, Introduction to Mechanics and Symmetry, vol. 17 of Texts in Applied Mathematics, Springer, New York, NY, USA, 1994.

[8] F. Lalonde and L. Polterovich, "Symplectic diffeomorphisms as isometries of Hofer's norm," Topology, vol. 36, no. 3, pp. 711-727, 1997.

[9] M. Bialy and L. Polterovich, "Geodesics of Hofer's metric on the group of Hamiltonian diffeomorphisms," Duke Mathematical Journal, vol. 76, no. 1, pp. 273-292, 1994.

[10] Y. M. Long, "Geodesics in the compactly supported Hamiltonian diffeomorphism group," Mathematische Zeitschrift, vol. 220, no. 2, pp. 279-294, 1995.

[11] F. Lalonde and D. McDuff, "Hofer's $L^{\infty}$-geometry: energy andstability of Hamiltonian flows, part I," Inventiones Mathematicae, vol. 122, no. 1, pp. 1-33, 1995.

[12] F. Lalonde and D. McDuff, "Hofer's $L^{\infty}$-geometry: energy and stability of Hamiltonian flows, part II," Inventiones Mathematicae, vol. 122, no. 1, pp. 35-69, 1995.

[13] Y.-G. Oh, "Chain level Floer theory and Hofer's geometry of the Hamiltonian diffeomorphism group," Asian Journal of Mathematics, vol. 6, no. 4, pp. 579-624, 2002.

[14] L. Polterovich, The Geometry of the Group of Symplectic Diffeomorphisms, Lectures in Mathematics ETH Zürich, Birkhäuser, Basel, Switzerland, 2001.

[15] A. Banyaga, The Structure of Classical Diffeomorphism Groups, vol. 400 of Mathematics and Its Applications, Kluwer Academic Publishers, Dordrecht, The Netherlands, 1997.

[16] J.-P. Ortega and T. S. Ratiu, Momentum Maps and Hamiltonian Reduction, vol. 222 of Progress in Mathematics, Birkhäuser, Boston, Mass, USA, 2004. 


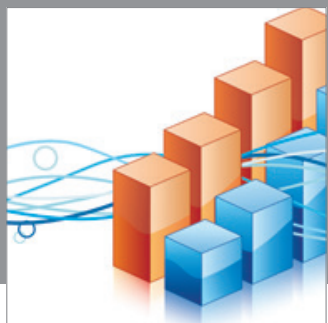

Advances in

Operations Research

mansans

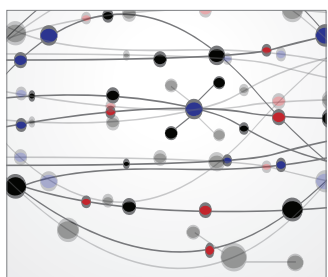

The Scientific World Journal
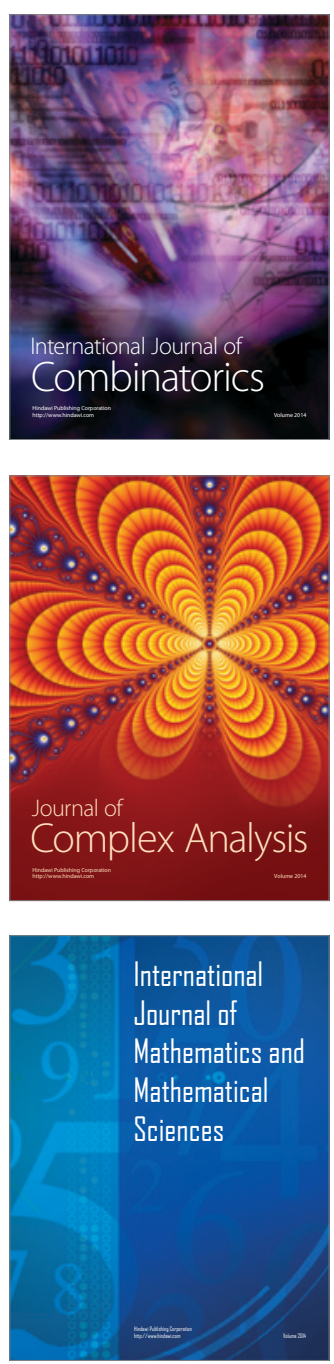
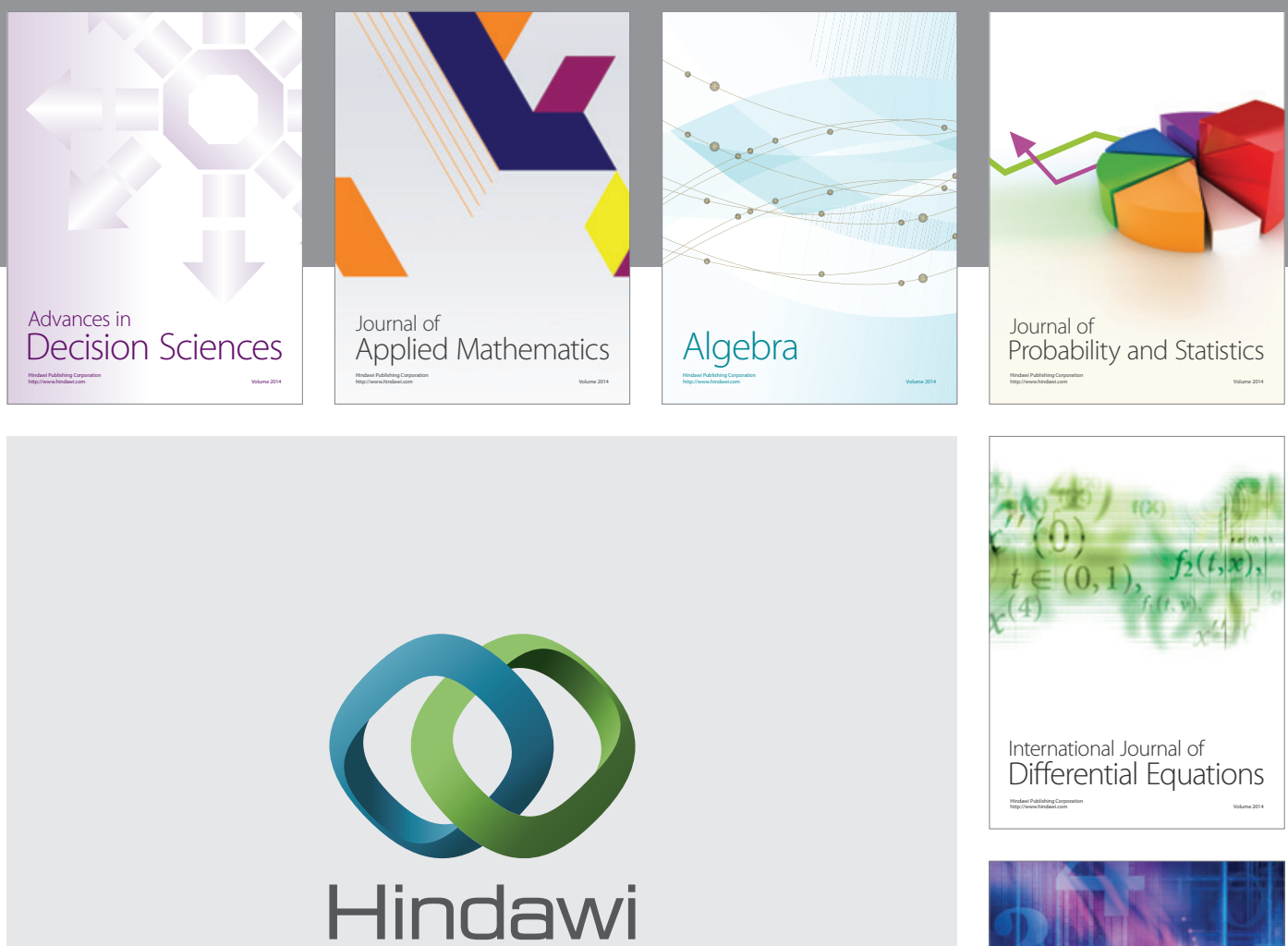

Submit your manuscripts at http://www.hindawi.com
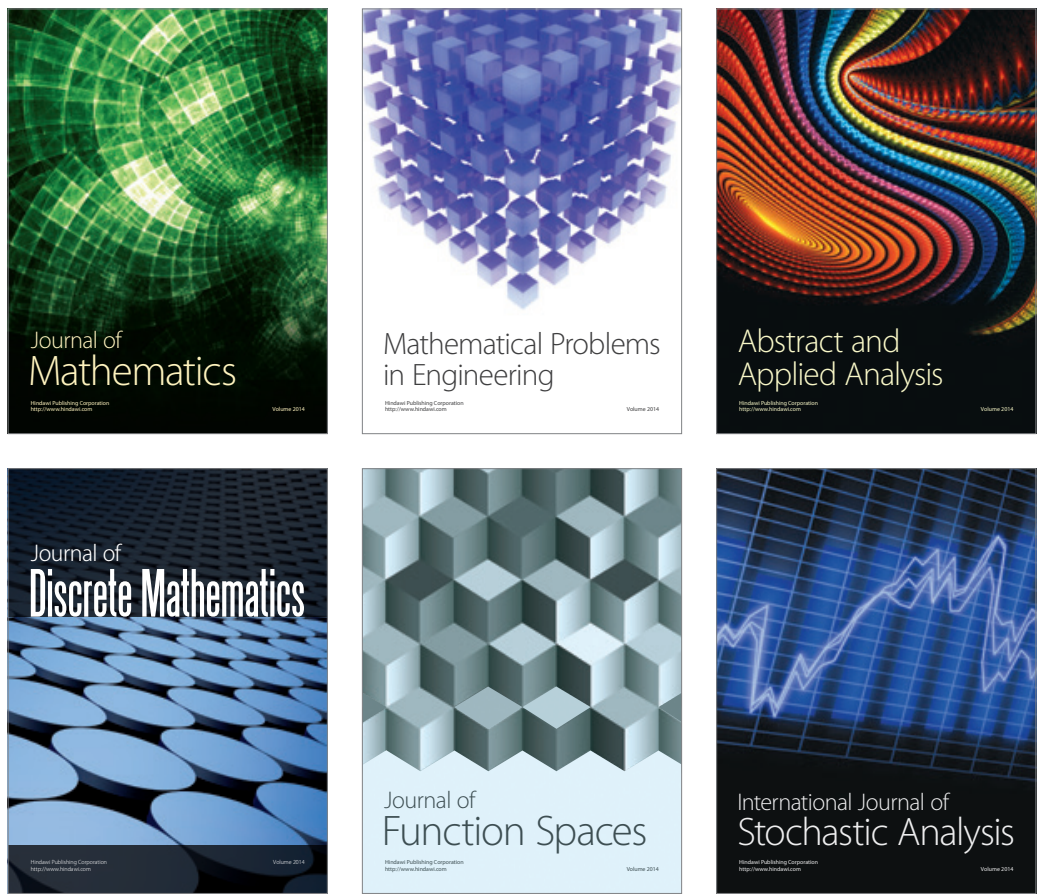

Journal of

Function Spaces

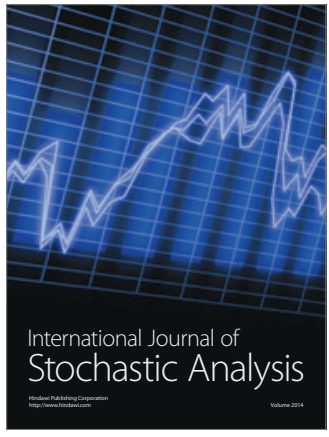

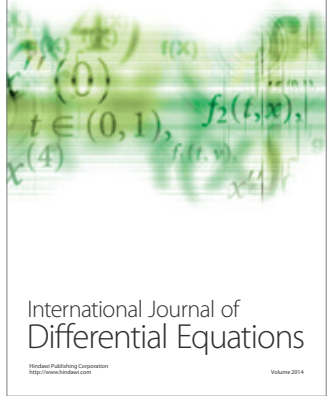
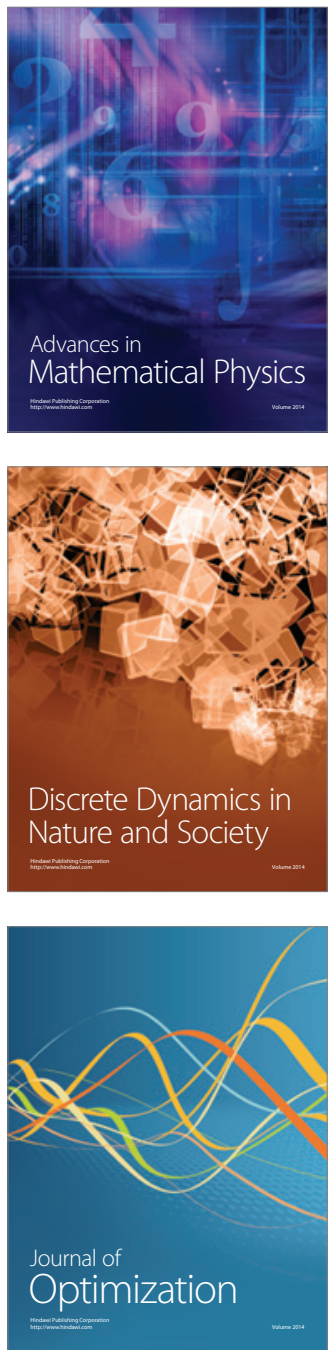